\title{
Topic 7 \\ Applications on High-Performance Computers
}

\author{
Jacek Kitowski, Andrzej M. Goscinski, Boleslaw K. Szymanski, and \\ Peter Luksch \\ Topic Chairs
}

The emergence of low-cost PC clusters together with the standardization of programming models (MPI and OpenMP) have paved the way for parallel computing to come into production use. In all domains of high performance computing, parallel execution routinely is considered as one of the major sources of performance. In some domains, like computational fluid dynamics, commercial codes already offer parallel execution as an option. In new domains, like bioinformatics, parallel execution is considered early in the design of algorithms and software. Besides clusters, grid computing is receiving increasing attention.

This year, 24 papers were submitted, 13 of which have been accepted (acceptance rate: $54.2 \%$ ), eight as regular papers, three as short papers. The topic is subdivided into three sections.

Numerical Analysis. Algorithms from linear algebra are considered in "Parallel Linear System Solution and its Application to Railway Power Network Simulation" and "Improving Performance of Hypermatrix Cholesky Factorization". "Implementation of Adaptive Control in Algorithms in Robot Manipulators using Parallel Computing" deals with robot manipulators whose dynamic parameters are not completely known. The paper "Cache Performance Optimizations for Parallel Lattice Boltzmann Codes" shows that efficient utilization of the memory hierarchy is essential to application performance.

Algorithms from other domains. Two papers address image processing: "Low Level Parallelization of Nonlinear Diffusion Filtering Algorithms for Cluster Computing Environments" and "Interactive Ray Tracing on Commodity PC". The paper "Comparing two Long Biological Sequences Using a DSM System" describes parallel implementations of the Smith-Waterman algorithm on a distributed shared memory system. "Parallel Agent-Based Simulation on a Cluster of workstation" presents a discrete time simulation implemented in a dynamically typed language. "Effectiveness of Parallelizing the ILOG-CPLEX Mixed Integer Optimizer in the PUBB2 Framework" describes the parallelization of branch and cut algorithms that solve mixed integer optimization problems on clusters of PCs.

Management of parameter studies and automatic optimization is addressed in three papers. "CAD-Grid: Corporate Wide Resource Sharing for Parameter Studies" describes a framework for corporate-wide shared use of simulation software and HPC resources that is being deployed in an industrial environment. A tool that manages execution of a large number of experiments, 
including resubmission of failed jobs, is presented in "Towards Automatic Management of Embarrassingly Parallel Applications". "Two Dimensional Airfoil Optimization using CFD in a Grid Computing Environment" describes a tool for automatic CFD optimization that assembles different industrial software components. 